\title{
Evaluación del sistema logístico de las empresas de transporte de pasajeros y envíos de mercancía de Norte de Santander, Colombia.
}

\section{Gustavo Enrique Bohórquez¹; José Sergio Fernández²; Mauricio García Mogollón ${ }^{3}$}

\footnotetext{
${ }^{1}$ Docente Universidad de Pamplona. Facultad de Ingenierías y Arquitectura, Programa de Ingeniería Industrial. Universidad de Pamplona,

${ }^{2}$ Docente Universidad Nacional del Chaco Austral,

${ }^{3}$ Docente Universidad de Pamplona. Facultad de Ciencias Económicas y Empresariales. Programa de Administración de Empresas. Universidad de Pamplona,
}

\section{Resumen}

En la presente investigación se busca demostrar la importancia que tiene la aplicación de la logística en las empresas de transporte y envíos de mercancías, siendo esta una excelente herramienta, que podría producir grandes ventajas competitivas, entre las cuales cabe mencionar, primero que nada la adecuación con base a la exigencia y necesidad del cliente, así como la mejora de la calidad en el servicio, abatiendo costos en todos los procesos, que tiene como efecto e impacto el poder ofrecer precios competitivos en pro de la satisfacción de los clientes. La investigación se llevó a cabo en el colectivo de las pequeñas cooperativas o empresas de transportes de pasajeros y envíos de mercancía, cuyas sedes están ubicadas en Colombia, departamento de Norte de Santander, principalmente en los municipios de Cúcuta, Ocaña y Pamplona. Se realizó bajo un enfoque de tipo cuantitativo, nivel descriptivo-proyectivo y diseño de campo, para lo cual se dividieron los elementos objetos de estudio en dos poblaciones: Población 1 cuya unidad de muestreo son los transportadores de pasajeros y mercancías y población 2 que corresponde a los clientes o usuarios del servicio de transporte de pasajeros y envíos de encomiendas. Después de analizar los resultados del trabajo de campo, se pudo concluir que las empresas no cuentan con procesos logísticos innovadores y estructuras físicas adecuadas para la prestación del servicio, como lo evidencia la ausencia de uso de técnicas logísticas como cross-docking, picking y paking, paletizaje, embalaje y trazabilidad, así mismo presentan una baja capacitación de personal y un deficiente confort, seguridad y calidad en servicio prestado. Sin embargo las empresas aun poseen un buen nivel de fidelidad por parte de los usuarios, 
lo que permite inferir que esta fidelidad responde más a la tradición de uso que a la satisfacción recibida.

Palabras clave: Sistema logístico, servicio al cliente, indicadores de gestión, distribución física de productos.

\section{Logistics System Assessment of companies transporting passengers and merchandise shipments in Norte de Santander, Colombia .}

\section{Abstract}

In the present investigation seeks to demonstrate the importance of the application of logistics in transport companies and freight shipments, this being an excellent tool, which could produce competitive advantages, among which include, first and foremost the adequacy based on the demand and customer needs and improving service quality, bringing down costs in all processes, which has the effect and impact the power to offer competitive prices towards customer satisfaction. The research was conducted in the group of small cooperatives or companies transport passengers and merchandise shipments, whose headquarters are located in Colombia, Norte de Santander department, mainly in the municipalities of Cucuta, Ocaña and Pamplona. It was conducted with a focus on quantitative, descriptive-projective level and field design, for which the elements objects of study were divided into two populations: Population 1 with a sampling unit are carriers of passengers and goods and population 2 corresponding customers or users of passenger service and parcel shipments. After analyzing the results of the field work, it was concluded that companies do not have innovative logistics processes and suitable for the service, as evidenced by the absence of use of logistics techniques such as cross-docking physical structures, picking and paking, palletising, packaging and traceability, also have low staff training and poor comfort, safety and quality in the service. However, companies still have a good level of loyalty from users, allowing infer that this fidelity responds more to the tradition of use to the satisfaction received.

Keywords: logistics system, customer service, management indicators, physical distribution.

*Para citar este artículo: Bohórquez GE; Fernández JS; García Mogollón M. Evaluación del sistema logístico de las empresas de transporte de pasajeros y envíos de mercancía de Norte de Santander,Colombia.. Revista Bistua.2016.14(1):125-130

+ Autor para el envió de correspondencia y la solicitud de las separatas: Gustavo Enrique Bohorquez. Docente Universidad de Pamplona. Facultad de Ingenierías y Arquitectura, Programa de Ingeniería Industrial. Universidad de Pamplona. email: gbohorquez@unipamplona.edu.co. 


\section{Introducción}

El intercambio económico del mundo globalizado de hoy, así como la constante innovación en los procesos productivos y en la prestación de los servicios que actualmente desarrollan las empresas en pro de competir en un mercado cada día más dinámico, se ha caracterizado por parte de las diferentes organizaciones en la necesidad del mejoramiento continuo de los procesos productivos, de manufactura y de los modelos gerenciales, empleados en la generación de bienes y servicios, que incidan positivamente en su entorno y que transciendan en el tiempo. En todo el mundo, las 24 horas al día, los 7 días a la semana, durante las 52 semanas al año, la logística se concentra en obtener y colocar productos y servicios donde se requieran, en el estado adecuado y en el momento preciso que se necesitan. La aplicación de una logística global es una estrategia diferenciadora que produce ventajas competitivas, en cuanto a la colocación de un producto o servicio en un mercado meta. Dos organizaciones pueden ofrecer un producto con características similares, pero aquella organización que pueda colocar ese producto en óptimas condiciones, en un menor tiempo posible, en el lugar adecuado y con un precio razonable, tendrá ventajas competitivas ante las demás ${ }^{1}$. Según Aljadi $^{2}$ existen tres empresas consideradas las más grandes del mundo con respecto al transporte de envíos y logística, en virtud del alto posicionamiento que tienen en el consumidor a nivel global en el servicio de entregas de mercancías, gracias a las estrategias agresivas que llevan en materia de mercado, finanzas y cadenas de distribución y abastecimiento. En primer lugar se encuentra la Federal Express, que emplea a más de 275000 personas y sus servicios llegan a más de 220 países, gracias a que posee la mayor flota aérea propia del mundo y además con la mayor capacidad de carga. En el segundo lugar se encuentra DHL, con cobertura en más de 200 países y una flota aérea de 420 aviones y 720000 vehículos y finalmente se encuentra United Parcel Service, que actualmente, cuenta con servicio aéreo y terrestre en más de 200 países de todo el mundo ${ }^{3}$. Asi mismo Jaramillo enuncia que a nivel de Colombia se destacan tres empresas con talla internacional como Transportadora Comercial Colombia, Servientrega S.A y Coordinadora Mercantil S.A, que apoyadas por plataformas satelitales, flotas de vehículos y aviones, y una red de servicios enfocado a las necesidades del cliente han logrado posicionarse dentro de las empresas más utilizadas a nivel nacional. El departamento de Norte de Santander, es un departamento que posee en la Ciudad de San José de Cúcuta una de las fronteras más calientes de toda la América del Sur, como lo es la frontera de San Antonio - Ureña - Cúcuta, por la cual de acuerdo a estadísticas emitidas por la Cámara de Comercio de Cúcuta $^{5}$, el departamento de Norte de Santander tuvo una balanza comercial por el orden de 95.624 .000 dólares, así 
mismo se destacó el número de pasajeros movilizados entre enero y marzo de 2011 que ascendió 172.874 .000 personas, con gran actividad turística y comercial en las ciudades de Ocaña y Pamplona. Toda esta actividad y flujo de usuarios ha ocasionado que se presente una demanda de propios y extranjeros por los servicios de transporte terrestre de pasajeros, el servicio de envió de paquetes, de encomiendas y documentos y el envío de giros de dinero dentro y fuera del departamento, servicios que son actualmente prestados por cooperativas y asociaciones de transportadores. Estas asociaciones registran un promedio de 2092.889.602 pesos, en activos, según lo enunciado por la Cámara de Comercio.

\section{Mètodo}

El nivel de la investigación que se propuso desarrollar fue descriptivo proyectivo $^{6}$, por cuanto busca explorar, describir, explicar y proponer alternativas de cambio a los problemas que enfrentan las empresas de transporte de pasajeros y envíos de mercancías de norte de Santander y que repercuten en la sociedad.Para el caso de la investigación realizada se consideró que el diseño de investigación fue de campo. Por cuanto los datos se recolectaron de la realidad por medio de cuestionarios que contenían una serie de ítems los cuales se aplicaron a las muestras de las poblaciones en estudio, siendo las mismas fuentes vivas, y donde los datos que se obtuvieron se consideraron de fuentes primarias ${ }^{7}$.
Con base en los objetivos de la investigación se consideró que el enfoque de la misma era cuantitativo, en virtud que estos estudios se asocian con los experimentos, las encuestas con preguntas cerradas o los estudios que emplean instrumentos de medición estandarizados, lo que permitió la posibilidad de generalizar los resultados más ampliamente ${ }^{8}$. El tipo de cuestionario, utilizado para las muestras fue directo, con preguntas cerradas, dicotómicas, de selección y escalas de tipo Likert ${ }^{9}$. Las poblaciones de estudio en la presente investigación se consideraron finitas y quedaron definidas como: población No 1 que corresponde a los transportadores de pasajeros y mercancías, según dato obtenido de la cámara de comercio de Norte de Santander, el sector cuenta con 77 empresas legalmente constituidas, población No 2 infinita, corresponde a los clientes o usuarios del servicio de transporte de pasajeros y envíos de encomiendas. Con un alcance espacial determinado por el Departamento de Norte de Santander, donde operan las empresas de transportes de pasajeros y envíos de mercancías, principalmente en Cúcuta, Pamplona y Ocaña, por ser estas sedes principales, quedando las muestras calculadas y definidas como muestra No 1 con un total de 43 empresas y por afijación proporcional será repartida de acuerdo a los estratos y la muestra No 2 para poblaciones infinitas, constituida por 96 clientes o usuarios del servicio. Dentro de las fases a desarrollar en la presente investigación se encuentra, realizar un diagnóstico del sistema 
logístico que actualmente implementan las empresas, para la prestación del servicio de transporte de pasajeros y envíos de encomiendas, de igual manera se buscó determinar el nivel satisfacción de los usuarios y clientes de las empresas de transporte de pasajeros y envíos de mercancías para que finalmente se pudieran identificar las brechas en el servicio prestado por las empresas de transporte de pasajeros y envíos de mercancías del departamento y así tener unos pilares en futuras propuestas de mejora en el sistema logístico de las organizaciones.

\section{Resultados y principales hallazgos}

Después de analizar los resultados estadísticos en cada población, se interpretaron con base en los objetivos planteados y en la significancia que tuvo la relación de las respuestas promediadas de las diferentes poblaciones enunciadas en el presente trabajo de investigación.

\section{Conclusiones}

1.-Con la ayuda del instrumento uno, se realizó un diagnóstico del sistema logístico de las empresas de transporte de pasajeros y envíos de mercancías del departamento, mediante el cual se pudo analizar dimensiones y variables importantes para conocer el estado actual de las organizaciones.

2.-El instrumento dos, se utilizó para recopilar y analizar información concerniente a determinar el nivel de satisfacción de los usuarios y clientes de las empresas de transporte, tomando como base de estudio las dimensiones, elementos tangibles, confiabilidad, capacidad de respuesta, seguridad y empatía ${ }^{10}$.

3.-Se identificaron las brechas en el servicio prestado por las empresas de transporte de pasajeros y envíos de mercancías del departamento, basado en las respuestas obtenidas por las poblaciones encuestadas, cuya información se tabuló y posteriormente se realizó un cálculo para determinar las brechas del sistema.

\section{Recomendaciones}

1.-Es importante que las empresas del sector, realicen la implementación de mejoras en el sistema logístico, cuyo propósito no es más, que, corregir las deficiencias en el servicio prestado y tener clientes totalmente satisfechos, generando así beneficios económicos en las empresas transportadoras.

2.-Realizar capacitaciones al personal en manejo del sistema logístico y atención al cliente, así como implementar el uso de indicadores que contribuyan a tomar las decisiones optimas en las empresas y mejorar la prestación del servicio.

3.-Implementar el uso de técnicas logísticas como cross-docking, paletizaje, embalaje, trazabilidad y picking y paking, siendo estos esenciales en los subsistemas logísticos de entrada, producción y salida.

4.-Realizar inversiones en tecnología, confort en vehículos y en el mejoramiento de las instalaciones, para que el usuario se sienta satisfecho al momento de tomar el servicio. 
130

5.-Por consiguiente es necesario que las empresas de transporte comiencen a tomar decisiones basadas en indicadores y empiecen a desarrollar sistemas que permitan desarrollar la logística a niveles competitivos a fin de reforzar el adecuado uso de la distribución física en pro del servicio al cliente, solo así las empresas podrán manejar las variables tiempo y espacio, como una ventaja competitiva.

\section{Referencias bibliogràficas}

1.- Casanovas A, Cuatrecasas L. Logística Empresarial. España: Gestión 2000; 2003.

2.- Aljadi D. Las 10 empresas más grandes del mundo. Revista Fortune. 2011; 1027: 2123.

3.- Inkpen A. Harvard Business Review. Southwesth Airlines. 2008; 0041: 16-18.

4.- Jaramillo J. Revista Dinero Transporte terrestre y aéreo en Colombia. 2008; 246: 910.

5.- Gonzalez J. Cámara de Comercio de Cúcuta. Boletín Informe de gestión. 2011; 031: 44-56.

6.- Hernandez R. Metodología de la investigación. Mexico: Mc Graw Hill; 2003.

7.- Hurtado J. Metodología de la investigación, una comprensión holística. Caracas: Quiron-Sypal; 2008.

8.- Balestrini R. Técnica de la investigación. Mexico: Mc Graw Hill; 1997.

9 Santesmases M. Diseño y análisis de encuestas. Madrid: Piramide; 1997.

Bistua Revista de la Facultad de Ciencias Basicas .Universidad de Pamplona. ISSN 0120-4211
Gustavo Enrique Bohorquez. Docente Universidad de Pamplona. Ingeniero industrial, Especialista en indicadores de gestión. Magister en gerencia de empresas.

Josè Sergio Fernandez. Docente Universidad Nacional del Chaco Austral, Ingeniero. Esp. en Ingeniería Gerencial, MSc. en Administración de Negocios. PhD. en Ingeniería.

Mauricio Garcia Mogollon. Docente Universidad de Pamplona. Administrador comercial y de sistemas. Magister en Administración, candidato a Doctor en Ciencias Gerenciales. 